and recall of the Good Samaritan patients. Grateful appreciation is also due to the volunteer members of the Mended Hearts Association of Boston, who assisted and participated in the study. Dr. Seymour Geisser and Dr. Peter Workman, Biometry Section, NIAMD, have assisted in the design of this investigation and in the analysis of data.

\section{REFERENCES}

Addis, G. J. (1959). Scot. med. 7., 4, 547.

Buckwalter, J. A., Naifeh, G. S., and Auer, J. E. (1962). Brit. med. F., 2, 1023 . Clarke, C. A., McConnell, R. B., and Sheppard, P. M. (1960). Ibid.,
1, 21.
Glynn, A. A., Glynn, L. E., and Holborow, E. J. (1956). Lancet, 2, 759. _- (1959). Brit. med. 7., 2, 266.

Glynn, L. E., and Holborow, E. J. (1952). 7. Path. Bact., 64, 775.

(1961) Arthr, and Rheum., 4, 203.

Kaklamanis, E., Holborow, E. J., and Glynn, L. E. (1964). Lancet, 1, 788.

Khattab, T. M., and Ismail, A. A. (1960). 9. Egypt. med. Ass., 43, 441. Maxted, G. R. (1940). Arch. Dis. Childh., 15, 181.

Nerell, G. (1963). Ann. hum. Genet., 27, 119.

Pham-Huu-Trung, Bessis, A., and Mozziconacci, P. (1961). Ann. Pédiat., $8,423$.

Roberts, J. A. F. (1957). Brit. 7. prev. soc. Med., 11, 107.

Roberts, J. A. F. (19. H., and Goslings, W. R. O.'(1963). Brit. med. F., 2,542 .

Walsh, R. J., and Kooptzoff, O. (1956). Aust. Ann. Med., 5, 17.

Wiener, A. S. (1962). Lancet, 1, 813.

Wilson, M. G. (1962). Advances in Rheumatic Fever, 1940-1961. Commonwealth Fund, Hoeber, New York.

\title{
Modes of Onset of Psychotic Depression
}

\author{
PETER HAYS,* M.B., B.S., D.P.M.
}

Brit. med. .., 1964, 2, 779-784

In this department of psychiatry depressions are classified into two principal groups, the main justification for this division being its value in treatment. In effect, with minor terminological differences between one doctor and another, our patients are categorized as having either reactive depressions, meaning comprehensible reactions of the personality to reverses or doleful circumstances, or, on the other hand, what might be called psychotic depressions. A recent presentation of old and new evidence (Kiloh and Garside, 1963) seems to confirm the usefulness of this common distinction. It is the psychotic depressions - the depressions judged likely to respond to electric convulsion treatment (E.C.T.) - that are studied here.

These psychotic depressions have certain clinical features that set them apart from the personality reactions called reactive depressions: for example, the mood is rather fixed, there are often the biological concomitants of depression such as loss of weight, loss of appetite, and constipation, and rather regularly one finds early-morning waking and diurnal variation, the patient feeling at his worst in the morning. One word often used to encompass these depressions is "endogenous"; a depression may be described as "endogenous in type" if obvious depression-producing factors (such as reserpine) operated, but the use of the term endogenous usually means that a syndrome has been recognized which points the way to electric treatment (or, in recent years, a drug with corresponding indications) and which implies that the mood is too fixed and the illness too set for psychotherapy and environmental amelioration to be appropriate as the main therapeutic methods.

\section{Material}

I examined the case summaries of " endogenously" depressed patients who had been at Atkinson Morley's Hospital in the years immediately before the antidepressive drugs came into use. If E.C.T. had been given I read the histories to see if an account of the manner in which the symptoms developed over a period of time was included, the presence of such an account constituting the criterion of selection.

Apart from the above patients, who constitute most of the material, any depressed patients presenting during late 1962 and early 1963 who were thought suitable for E.C.T. were also

\footnotetext{
- Senior Lecturer in Psychiatry, St. George's Hospital, London.
}

seen, and accounts were obtained of the mode of onset of their illnesses, provided that the mode of onset had not been disturbed by the use of antidepressive drugs.

The work fell into four phases, the early results leading to further explorations, and it is convenient to present the methods and results in a correspondingly consecutive manner.

\section{Method 1}

Dr. J. A. Fraser Roberts kindly advised me at this stage; he suggested that I map the mode of development of the symptoms in a graphic form, offering at the same time guidance about scoring and presentation.

Essentially a patient's clinical state at a given point in time was represented by a histogram. On the histogram a set of symptoms was recorded, with some indication of the severity of each symptom. Such histograms were constructed for the syndrome at the time of presentation and for each threemonthly period preceding this. In this way a series of histograms was available for each patient, chronologically arranged, and together giving a graphic representation of the manner in which the illness had developed.

The symptoms considered are shown in Fig. 1, and are those commonly or frequently present in psychotic depressions. It will be seen that symptoms are roughly grouped, those in black being thought most characteristic of the syndrome and those on the right (hatched) being symptoms which, though commonly enough found in psychotic depression, are not in themselves diagnostic of the state; indeed, these last symptoms (anxiety, symptoms increasing towards evening, and difficulty

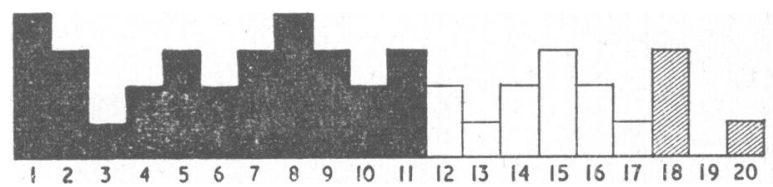

FIG. 1.-Key :-1: Suicidal ideas. 2: Depression. 3 : Retardation. 4: Self-reproach. 5: Worse in mornings. 6: Early waking. 7: Indecision. 8: Loss of reactivity. 9: Loss of interest 10: Loss of energy 11: Loss of concentration 12. Los 12: Loss weight. 15: General somatic symptoms. 16: Gastro-intestinal symptoms. 17: Obsessional symptoms. $18:$ Anxiety.
19: Worse in evenings. 20: Difficulty in falling asleep. 
in getting off to sleep), taken alone, are those of a neurotic rather than a psychotic condition; this grouping reflects an interest taken in the connexion, if any, between neurotic and psychotic symptoms, and is referred to later, when the results of the investigation are presented.

Scoring of individual symptoms was based on sets of operational definitions constructed early in the project, but when old case-notes were being utilized the scoring was of ten approximate and in many instances incomplete. Because of its length the complete list of definitions is not published here, though cyclostyled copies are being prepared and will be sent on request to colleagues wishing to criticize or repeat aspects of the work: by way of illustration the item named suicidal ideas scored 1 if the patient thought life was not worth living or had occasional thoughts of suicide or oblivion ; 2 if these morbid thoughts were frequent or if, despite infrequency of suicidal thoughts, the method was worked out or the patient would positively have welcomed death ; 3 for suicidal ideas definite and repeated with the method worked out ; and 4 for an attempt.

When the data were assembled and transcribed in graphic form, then, each patient had a series of histograms in chronological order on a large sheet of paper. A total of 81 patients were worked up in this manner.

I then leafed through the 81 sheets repeatedly, looking for purely visual patterns.

\section{Results}

Four such patterns presented themselves, and examples are shown in Fig. 2.
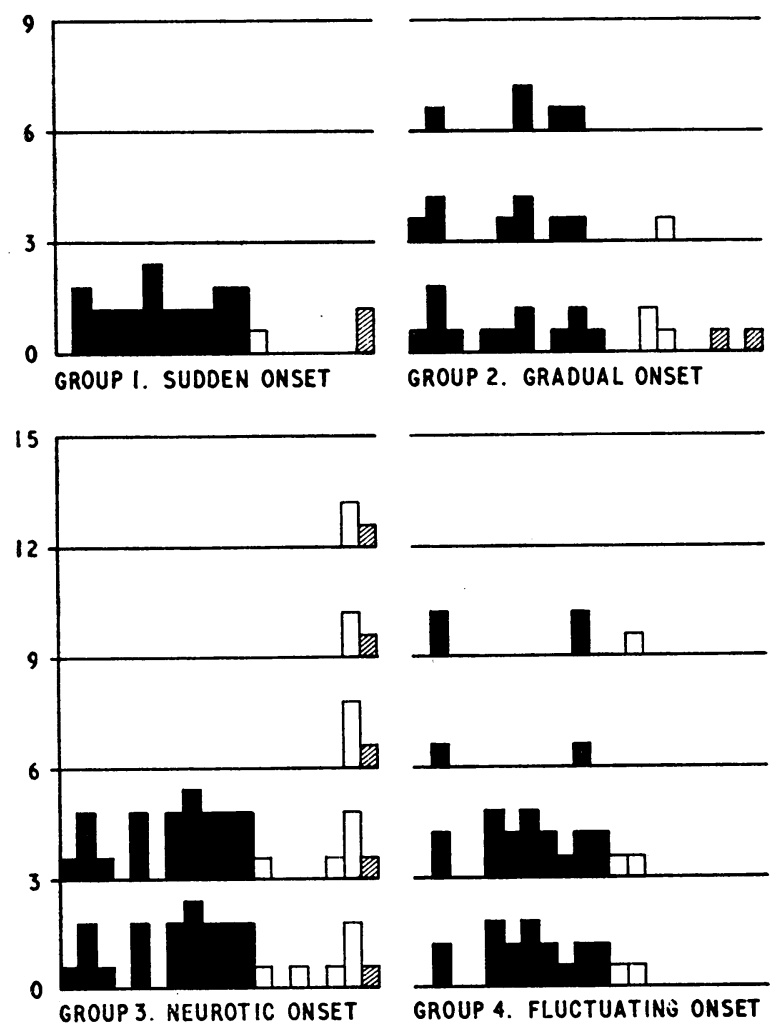

FIG. 2.-The four patterns of development of psychotic depression. The numbers refer to the number of months before presentation.

The first pattern is perhaps the closest to the classical and expected, in which the symptoms develop suddenly, almost explosively, out of a clear sky. The second, the commonest in this series, represents an illness in which the symptoms are depressive from the start but develop only gradually, taking several or many months to reach the maximum level for the individual. The third represents an illness in which the initial symptoms are predominantly those of anxiety and its elaborations, with depressive symptoms supervening at a later stage, the graphic impression here consisting of a shift to the left. The fourth pattern is seen comparatively seldom: the symptoms fluctuate considerably before reaching their full force.

Mutually exclusive operational definitions for these patterns were devised and the 81 patients were subdivided accordingly: group 1 (sudden onset), 11 ; group 2 (gradual onset), 50 ; group 3 (neurotic onset), 14 ; group 4 (fluctuating onset), 6.

\section{Comment}

Evidently the question was whether these groupings were merely forced subdivisions or whether they represented some real clinical distinction-that is to say, whether other notable differences could be found in the characteristics of the illnesses or the backgrounds of the various groups of patients.

\section{Method 2}

The original numerically scored sheets, separate from the histograms, were used for further scoring. Several items were considered, of which only a few produced scores that were large enough in aggregate to be useful in statistical comparison and thus worth recording here. Also, the stage of numerical manipulation having been reached, the group of illnesses with fluctuating onsets (group 4) was reckoned too small to allow any conclusions to be drawn about common factors, and, though it was scored and the scores were inspected, it is not considered further.

Except where otherwise stated the t-test was applied in the assessment of significance.

\section{Results}

Of the patients in groups 1,2, and 3,38 out of 75 were male, this fraction reflecting the symmetry of the department. Of the males, 22 out of $38(58 \%)$ were in group 2, while 28 out of 37 $(76 \%)$ of the females fell into this group.

The average ages of the groups were as follow: group 1, 47 years; group 2, 48 ; and group 3, 48.

Anxiety-producing Stress.-This is difficult to assess and the measure was one of the least satisfactory ; however, an attempt was made to score any anxiety-producing stress undergone by these patients at the time their earliest psychiatric symptoms started. In aggregate there seemed to be a tendency for those with illnesses of neurotic onset (group 3) to have undergone rather more in the way of worrying stresses than those in the other groups, but the differences were not significant.

Number of E.C.T.s.-The elimination of patients who, for various reasons, had not completed their courses made for a reduction in numbers, but this measure, by contrast with the first, was one that could be scored precisely. Group 1, the sudden-onset group, needed an average of 5.4 E.C.T.s ; group 3, whose illnesses started with anxiety symptoms, needed significantly more-an average of $7.0 \quad(\mathrm{P}<0.05)$. Group 2, the "gradual-depressive"-onset group, was intermediate with an average of 6.1 E.C.T.s: differences between groups 1 and 2, and between groups 2 and 3 were not significant.

Constitutional Predisposition.-Past affective illnesses, family history of affective illnesses, and evidence of cyclothymia contributed to this score. Group 1, who descended most steeply from an asymptomatic state to one of psychotic depression, had the highest constitutional predisposition. Group 3, with perhaps the furthest from what might be regarded as the classical mode of onset, had the least constitutional predisposition. Group 2 was intermediate. Differences between group 1 and each of the other groups ( 2 and 3 ) were highly significant $(1: 3$, 
$\mathrm{P}<0.001 ; 1: 2, \mathrm{P}<0.005)$; differences between groups 2 and 3 were not significant. Evidence of hypomanic episodes or cyclothymia was found in 4 out of 11 in group 1 and in 2 out of 50 in group 2 . Only 1 out of 14 in group 3 was doubtfully thought to have had a period of excitement in the past.

Stresses Other than Anxiety-producing Stress-"Depressive" Stress.-I include in this measure bereavement, reserpine, childbirth, the menopause, and influenza-like illnesses: all of these are known to be associated in some way with the advent of psychotic depression and all of them may be more or less objectively scored. They either were or were not operating at the start of the illness. It transpired that it was comparatively uncommon for such stresses to operate in the sudden-onset depressions of group 1, and comparatively common for these stresses to operate in the other depressions, whether these started with mild depressive symptoms (group 2) or neurotic symptoms (group 3). The difference between group 1 and each of the other groups was significant $(1: 2, \mathrm{P}<0.005 ; 1: 3, \mathrm{P}<0.05)$. It may be said that depressions of sudden onset are mainly made up of illnesses in which no cause is discernible ; and that, conversely, the gradual-onset and neurotic-onset depressions are mainly made up of illnesses in which some known, objectively recognizable factor operated at the beginning of the illness.

\section{Comment}

The low incidence of causal factors in group 1 and the high incidence in groups 2 and 3 might be taken to indicate that a clinical distinction (based on the mode of onset) was being drawn between a group of endogenous and a group of exogenous psychoses. Certainly, despite authoritative pronouncements about the unity of psychotic depressive illnesses, some of the figures obtained from this investigation appeared to set the sudden-onset psychotic depressions off from the rest.

If this distinction were biologically founded the genetics and constitutional bases of the groups should differ. As already noted, the sudden-onset group 1 differs quantitatively in that a much higher loading of affective illness is to be found in the patients' backgrounds. Although the differences are great, this merely amounts to saying that the more predisposition a patient has the steeper the gradient in the development of his illness, and thus the finding is entirely compatible with the traditional proposition that all psychotic depressions are strung along a continuum, with many factors combining to determine the individual patterns shown.

However, if a real distinction between endogenous and exogenous psychotic depressions can be drawn, patients with sudden-onset depressions and high constitutional loading should not have correspondingly high past incidences of menopausal, post-influenzal, puerperal, bereavement, or reserpine depressions, or family histories of such apparently exogenous psychotic depressions. On the other hand, if psychotic depressive illnesses are really all much of a muchness, the high constitutional loading for affective illnesses shown by the sudden-onset group 1 should be reflected in a similar high loading of depressions triggered off by such readily available stresses as the menopause and bereavement.

Considerations of this nature led to the use of the next measure.

\section{Method 3}

Individuals were scored for their past medical histories and family histories of menopausal, post-influenzal, puerperal, bereavement, and reserpine depressions.

\section{Results}

More of these seemingly exogenous depressions were found in the backgrounds of those patients with a gradual onset to their illnesses (groups 2 and 3 ) than in those in group $1(\mathrm{P}<0.1)$.

\section{Comment}

Taken on their own, the differences were not statistically significant. In the everyday meaning of the word, however, the significance of the finding was enhanced by comparing it with the difference in total affective constitutional predisposition between those groups, which was not only more marked-for example, $1: 2, \mathrm{P}>0.005$-but was entirely the other way. On the evidence of this reversal of pattern the implication seems to be that group 1 and groups 2 and 3, though they display the same phenomena at the heights of their illnesses, are otherwise not closely related.

\section{Gradual-onset and Neurotic-onset Depressions}

So far in this account I have concentrated on the differences between depressions of sudden onset and those of more gradual onset because these differences were the most pronounced. Consideration of differences between the two groups of gradually developing psychotic depressions-that is, between those starting with mild depressive symptoms (group 2) and those starting with anxiety symptoms unmixed with depressive symptoms (group 3)-shows that these are for the most part only suggestive.

In one instance, mentioned only in passing here, when the scores for a past history of neurotic illness or a premorbid personality thought to be notably anxious or obsessional were compared, group 3 scored significantly higher than group 2. This finding was mundane, and entirely congruous with a hypothesis that the two gradual-onset groups were closely connected. Furthermore, after so many examinations of patterns of onset, I had come to feel that the two types of illness were at least interwoven ; for example, I noticed that an influenza-like illness afflicting a patient without pre-existing symptoms led to a much more gradual depressive illness than a corresponding virus illness affecting a patient with pre-existing overt anxiety or obsessional symptoms, in whom the evolution could be rapid, as if the anxiety and the virus illness were additive in their effect. Again, anxiety-producing stress seemed to lead to depressive symptoms from the start in some patients and to anxiety symptoms in others, while such "depressive" stresses as the menopause and influenza-like illnesses could lead to initial symptoms that were either those of anxiety (group 3) or those of depression (group 2).

Impressions of this kind, though probably worth reporting, are of little value when an attempt is being made to demonstrate whether subdivision of the more gradually developing depressions is justifiable and useful. Probably some significant differences could have been extracted from various combinations of the scores obtained, but the scoring methods used, in many instances on old case histories, may credibly be described as crude ; and I thought that further manipulation of these figures was probably not justifiable except for the purpose of guiding further research.

Fortunately, confirmation or denial of the existence of real distinction between groups 2 and 3 could be obtained without reference to the scores reported so far, since another series of depressed patients was being studied in the department at the same time.

\section{Method 4}

Dr. Peter Richmond and Dr. A. H. Roberts had been conducting a blind comparative trial of antidepressive drugs on depressed out-patients, the results of which have been reported in detail elsewhere (in press). These patients were divided into a "reactive" group and what was called an "endogenous" group, meaning illnesses that correspond precisely in quality with the subject of the present study.

Dr. Richmond kindly offered me full use of his then incomplete data. I looked through the case notes of the 
patients concerned in the trial, selecting those with psychoticthat is, "endogenous"- -depressions. I then tried to classify these patients into neurotic-onset and depressive-onset groups; this endeavour was hampered by the laconic nature of the outpatient notes (some of them my own) and their concentration on the phenomena displayed at the time of presentation rather than the history of the illness from the moment when symptoms started, but it was possible to estimate the mode of onset of 53 gradually developing depressions ; 31 being thought gradualdepressive (group 2) and 22 neurotic-onset (group 3). The names of the patients were listed under two uninformative headings and the list was given to Dr. Richmond.

An assumption was made that these two groups of patients corresponded to groups 2 and 3 of the main study. The hypothesis was that they differed in certain essentials; and the prediction was that they would respond differently to antidepressive drugs.

At the end of the drug trial Dr. Richmond noted against each name whether or not a "definite" drug response had occurred, together with the name of the drug successfully used, and returned the list.

\section{Results}

Of the depressive-onset group 21 out of 31 (68\%) made a definite response to one or other drug. Of the anxiety-onset group 8 out of $22(36 \%)$ made a definite response. The difference is obvious on inspection and may be demonstrated by $\chi^{2}$.

A review of the patients included in the list led to the exclusion of nine thought by Dr. Richmond to be " doubtfully endogenous" or essentially reactive but did not materially alter the proportions just shown, though the reduced number of patients altered the $P$ value (nevertheless leaving it $<0.05$ ).

No particular drug could be singled out as relatively more or less effective in the neurotic-onset group than in the gradualdepressive-onset group.

\section{Comment}

The wide applicability of E.C.T. means that this treatment is of comparatively little use as a diagnostic test when a functional psychosis lies symptomatologically in the borderlands between two supposed diagnostic entities. The antidepressive drugs, however, seem more specific than E.C.T. in that, though they too find their main application in depressive states, they are seldom of use and are often contraindicated in illnesses for which E.C.T. is readily invoked (schizophrenia and hypomania, for example).

The advent of antidepressive drugs brought with it the hope that they might be helpful in classifying depressive illnesses. To some extent this hope has been borne out in that the success of these drugs in patients with the psychotic (" endogenous") depressive syndrome has served to confirm that these states differ essentially from the reactive depressions. Having determined that the prime indication for, say, imipramine is psychotic depression, further attempts have been made to find pointers which may be used to predict success for the drug. Most analyses largely confine themselves to the phenomenology of the syndrome at the time of presentation, and a typical finding is that success with imipramine correlates significantly with early-morning waking (Kiloh et al., 1962). Since earlymorning waking correlates also with the psychotic depressive syndrome such a pointer introduces an element of circularity and simply confirms that the more closely a depressive syndrome conforms to the typical pattern the more likely it is to respond to drug therapy.

Even typical psychotic depressions respond uncertainly to antidepressive drugs; published results vary somewhat, but Dr.
Richmond's finding that about $50 \%$ of his "endogenously" depressed patients were "drug successes" is accepted here as being the norm.

When a group of psychotically depressed patients may be subdivided into two differently reacting moieties by the application of the sole criterion of the mode of onset of the illness it is obviously permissible to speculate that here too a clinical distinction is being drawn between illnesses which, though they may be interconnected in some degree, deserve separate identities in any diagnostic classification.

\section{Conclusions}

The evidence presented here does not accord with the supposition, hallowed in textbooks, classical accounts, and statistically inspired nosological systems, that all affective psychoses, with the possible exception of involutional depressions, can be regarded as variants of the manic-depressive psychosis.

Instead, the syndrome of psychotic depression seems to constitute a phenomenologically stereotyped end-state with a variety of sets of constitutional and aetiological antecedents. Application of the single criterion of mode of onset to an otherwise apparently undifferentiated group of psychotic depressions enables separate illnesses to be singled out. Because of the positive nature of the present evidence a review of the necessarily negative evidence adduced when the unity of affective psychoses is upheld is not offered here. The apparent subdivisions of the syndrome are as follows.

\section{Sudden-onset Depressions}

The syndrome of psychotic depression develops in a few weeks, or less, the patient having been symptom-free during the immediately preceding period. No cause for the depression is discernible. The patient's past medical history, family history, and premorbid personality contain indications of considerable constitutional predisposition to unprecipitated depression, and sometimes evidence of hypomanic episodes or cyclothymia.

Rennie (1942) recorded an abrupt onset in $12 \%$ of his large series of affective psychoses and noted that these were chiefly confined to the cyclothymic cases, though he did not use the finding for the purposes of diagnostic subdivision, concluding that "the manic-depressive illness $[$ sic $] \ldots$ appears to be one of gradual onset."

Patients suffering from sudden-onset psychotic depressions may thus be said to be in the depressed phase of true manicdepressive psychosis, and their illnesses are truly endogenous. The condition is classical and these patients have been intensively studied (most of the observations about pyknic habitus, extraversion, etc., probably referring to this circumscribed group).

\section{Gradual-onset Depressions}

The syndrome of psychotic depression develops gradually: from an asymptomatic state the patient takes longer than six weeks, and commonly takes several months, to reach a severely depressed state. There is no evidence of constitutional predisposition to hypomanic or manic episodes and the patient is not cyclothymic. Often, however, there is evidence of constitutional predisposition to psychotic depressions and, commonly, previous depressions or depressions occurring in near relatives have been associated with exogenous factors. Commonly also, exogenous factors, such as the menopause, reserpine, childbirth, influenza-like illnesses, or bereavement, operated at the time of 
onset of the first mild psychiatric symptoms, though the ultimate evolution of these depressive symptoms into the full syndrome of psychotic depression may be unaccompanied, even when the deterioration is rather rapid and takes place after some delay, by any discernible stress.

Some other studies support the separate identity of this syndrome: for example, Leonhard et al. (1962) found that manic-depression was genetically distinct from "melancholia" and "depression" (manic-depressives having very much more evidence of affective illness in their family histories).

The exogenous nature of many depressions was asserted by Rennie (1942), who found "definitely disturbing life situations" in $79 \%$ of 208 depressed patients ; this conclusion has been criticized because the stresses cited antedated the onset of frank depression by several months, but the validity of this criticism is lessened or annulled if the syndrome is recognized as being one of slow and inexorable development.

Patients suffering from gradual-onset psychotic depressions constitute the majority of the so-called cases of "endogenous" depression seen in in-patient practice and the great majority of those seen in out-patient clinics. The majority display exogenous factors at the start of the illness, so that it may be said that where the onset is insidious it is sensible to try to date the beginning of ill-health and to seek and to pay due regard to stresses experienced at that time. It would seem appropriate to follow the Scandinavian practice and refer to these illnesses as reactive psychotic depressions but for the implication in the phrase that the stress occasioning the reaction is psychological, which it may but need not be ; whether they are called melancholias, exogenous psychotic depressions, or gradual-onset depressions probably matters less than does abandoning the term "endogenous" in this context.

Both the menopausal epoch (identified by the cessation of menstruation and here delimited by the duration of hot flushes) and reserpine medication are relatively long-lasting stresses. The clearest examples of gradual-onset depressive illnesses are therefore to be found in association with the other common "depressive" stresses-bereavement, influenza-like illnesses, and childbirth. The duration of these stresses is short or, in the case of bereavement when the true duration is hard to calculate, the stress is maximal at the start. Commonly, nevertheless, the depressive illness that supervenes does so only gradually, provided that overt psychiatric symptoms do not antedate the stress under consideration.

\section{Neurotic-onset Depressions}

The syndrome of psychotic depression is here preceded by a state, phenomenologically indistinguishable from a neurotic state, in which anxiety and its elaborations form the central symptoms, without evidence either of depression, the psychotic diurnal rhythm, or the general lowering of output, initiative, powers of concentration, and so on that characterize the mature illness.

Like the gradual-onset illnesses just described, these illnesses differ from the sudden-onset-that is, probably true manicdepressive-illnesses in that the demonstrable constitutional predisposition is much less (with hypomanic episodes not found), and exogenous factors commonly operate at the time of onset of the symptoms.

The differences between the neurotic-onset patients presently being described and the gradual-onset group are for the most part interesting rather than striking, so that the main justification for formally separating the groups is the finding, via a parallel series of patients, that the gradual-onset group responded better to antidepressive drugs than did the neuroticonset group. Nevertheless, the points of difference-including a greater tendency on the part of the anxiety-onset group to undergo anxiety-producing stress at the start of the illness and to have evidence of past neuroticism, and a lesser tendency to have a family history of depression or to have "depressive" stresses (with the exception of influenza-like illnesses, which occurred slightly more commonly)-are intriguing. Being unwilling to compound the sin of introducing yet another classification of depressions, I shall not speculate about the possible relationships that might emerge if a larger number of patients were analysed: however, there are already indications that orderliness could be introduced to the point where, for example, what is symptomatically an "anxiety state" could be identified as either a neurotic condition or the start of a psychotic depression (with concomitant guidance about treatment and prognosis), and further work is under way.

Fitting these neurotic-onset psychotic depressions into existing nomenclatures is made more difficult by the fact that it is just these illnesses, lying as they do between the " endogenous" complex of illnesses and the reactive (personality reaction) depressions, that make the exceptions when this oversimplified nosological dichotomy is imposed on a sample of depressed patients. They might suitably be called neuroticonset depressions until further analyses clarify their status.

Examples occur frequently in normal practice: the patient with classical obsessive-compulsive neurosis who ultimately develops deep depression and receives E.C.T. for this element of the symptomatology; the phobic housewife who struggles along in some sort of equilibrium until depression, reduced initiative, and loss of hope for the future make admission necessary ; and the patient who has an anxiety state that presents no psychodynamic anomalies but who, in the course of psychotherapy; becomes depressed and starts to display the symptoms and diurnal rhythm of psychotic depression-these are all members of this group. The fact that the onset is neurotic, however, does not necessarily mean that the causation is predominantly psychological ; and, indeed, a preliminary impression from further work being done on this topic is that where anxiety symptoms that herald a psychotic depression cannot be adequately explained psychodynamically they may often be traced to a recent influenza-like illness.

This classification is not definitive or even entirely comprehensive. Involutional depression was not specially studied: three patients to whom this label had been appended fell into the gradual-onset and neurotic-onset groups ( 2 and 3 ), and each was thought to have an exogenous illness, but " involutional depression" is diagnosed inconsistently and sometimes casually in the department, so that not even tentative conclusions about the status of this diagnosis can be drawn. Furthermore, I have known patients, usually bereaved, whose first illnesses were gradual in onset but who later had depressive illnesses that were sudden in onset and apparently unprecipitated. It might be argued that some of these patients were not truly asymptomatic between their first depression and the next ; or that, the first depression having been treated, the second episode was more in the nature of a relapse than a recurrence ; or, as I think most probable, that the initial exogenous depression sensitized the patient in some way, rendering further illnesses much more likely. But, however the case is put, the existence of such exceptions must detract from the reliability of any classification based solely on the mode of onset.

Nevertheless, if these results and conclusions are accepted and broadly confirmed, it follows that any clinical workers selecting a small group of depressions for diagnostic uniformity would be prudent to take the mode of onset into consideration. In the same way, categorization of more widespread groups according to the mode of onset might be revealing in the analysis of results obtained, whether these are concerned with sociological, biochemical, epidemiological, psychological, genetic, or therapeutic matters. Such work, now done on an increasingly large scale, loses much of its value if the task of diagnosis has not been completed, and indeed might be said to be premature. 


\section{Summary}

Eighty-one psychotic ("endogenous") depressions were studied to determine the mode of onset of the symptoms, using a graphic method. Four patterns emerged from this investigation: (1) sudden onset (11); (2) gradual onset (50); (3) neurotic onset (14); and (4) fluctuating onset (6).

Groups 1,2, and 3 were examined further to see if other differences could be found. The results seemed to indicate that group 1 (sudden onset) comprised illnesses that were separate and distinct from those of groups 2 and 3, corresponded to the depressed phase of manic-depressive psychosis, and were truly endogenous; while the depressions of groups 2 and 3 were mainly exogenous, and, though probably related to each other, showed different responses to antidepressive drugs, group 2 responding more consistently.
A classification of psychotic depressive illnesses, based on the evidence presented, is set out.

I am grateful to Professor D. Curran and to Dr. J. A. Fraser Roberts for encouragement and detailed advice ; to Mr. H. Gwynne Jones for guidance in the application of statistical methods; to the consultants at St. George's Hospital for access to patients under their care ; to Dr. P. W. Richmond and Dr. A. H. Roberts for their co-operation and help; and to Mrs. J. Haigh for drawing the histograms.

\section{REFERENCES}

Kiloh, L. G., Ball, J. R. B., and Garside, R. F. (1962). Brit. med. F., $1,1225$. and Garside, R. F. (1963). Brit. F. Psychiat., 109, 451.

Leonhard, K., Korff, I., and Schulz, H. (1962). Psychiat. et Neurol. (Basel), 143, 416 .

Rennie, T. A. C. (1942). Amer. F. Psychiat., 98, 801.

\title{
Effects of Surgery under General Anaesthesia on the Electrocardiogram in Ischaemic Heart Disease and Hypertension
}

\author{
D. A. CHAMBERLAIN, ${ }^{*}$ M.B., M.R.C.P.; J. EDMONDS-SEAL,* M.B., F.F.A. R.C.S.
}

\section{Brit. med. F., 1964, 2, 784-787}

Modern agents and techniques have made surgery under general anaesthesia comparatively safe, and there are now few contraindications to its use. Nevertheless, there are patients in whom it carries an appreciable risk; the significance of recent myocardial infarction in this respect is well recognized and operative mortality has been shown to be particularly high in the two years following an acute episode (Knapp et al., 1962). Less emphasis has been placed on the dangers in chronic ischaemic heart disease. Etsten and Proger (1955) found, in a large series, a mortality of $2.9 \%$ in asymptomatic patients with electrocardiographic evidence of coronary heart disease, compared with $2 \%$ in those with normal electrocardiograms (E.C.G.s). In a similar study, comparing patients with and without arteriosclerotic heart disease, Nachlas et al. (1961) noted a much greater difference, with mortality figures of $10.5 \%$ and $3.5 \%$ respectively. Driscoll et al. (1961) recorded E.C.G.s in 496 patients before and after surgery. They found marked fresh ischaemic changes post-operatively in $23 \%$ of the 145 patients in the series with chronic coronary heart disease, hypertension, diabetes, or abnormal pre-operative E.C.G.s; similar changes occurred in less than $3 \%$ of the remaining 351 patients without these conditions.

In the present series the changes which occurred postoperatively in the E.C.G.s of patients with ischaemic heart disease and hypertension have been studied. By relating them to the types of surgery and anaesthesia employed, an attempt has been made to identify those factors which are particularly likely to be associated with electrocardiographic deterioration.

\section{Material and Methods}

Conventional 12-lead E.C.G.s were taken pre-operatively in patients with known or suspected ischaemic heart disease, widespread arterial disease, or hypertension which, for the purpose of this study, was defined as a systolic pressure of $200 \mathrm{~mm}$. $\mathrm{Hg}$ and over or a diastolic pressure of $120 \mathrm{~mm}$. $\mathrm{Hg}$ and over on

- From the Department of Cardiology and the Department of Anaesthesia, St. Bartholomew's Hospital, London. routine ward sphygmomanometry. Arrangements were made before surgery for a tracing to be taken the day after operation from those with definite ischaemic heart disease and from those who were hypertensive by the criteria above. Where the postoperative E.C.G. was markedly changed compared with the previous one, further tracings were usually taken three days later. There were 217 sets of tracings available for analysis; of these, all but 12 were judged to be ischaemic, or had ST and $T$ changes of left ventricular strain.

Patients undergoing thoracic surgery were not accepted into the series. Those with left bundle-branch block were excluded because of the difficulty in detecting fresh ischaemia from their tracings, as were patients in whom surgical dressings made a conventional 12-lead E.C.G. impossible.

Information was obtained from the patients' notes and anaesthetic record cards on the types of operations, the anaesthetic methods and agents used, and in most cases on the bloodpressure measurements throughout surgery.

The pre-operative and post-operative E.C.G.s were reported in pairs without knowledge of the order in which they were taken, and any changes were noted. For simplicity, the changes were classified in the following manner: grade 0 , no change ; grade 1 , slight changes of a type and a degree which are commonly seen in day-to-day variations in patients with ischaemic heart disease ; grade 2, marked changes in ST segments or $\mathrm{T}$ waves, or the development of bundle-branch block; grade 3 , changes in $T$ waves of a degree sufficient to suggest fresh infarction.

There were a few E.C.G.s which were very abnormal before and after surgery, causing difficulty in assessing the significance of $\mathrm{T}$-wave changes. However, the reporting of most pairs of tracings presented no great problem.

\section{Results}

Of the 217 sets of E.C.G.s analysed (Table I), 48 (22\%) developed significant electrocardiographic deterioration post- 